\title{
Low Power Mixed-Mode BIST Based on Mask Pattern Generation Using Dual LFSR Re-seeding
}

\author{
Paul M. Rosinger* and Bashir M. Al-Hashimi \\ Dept. of ECS \\ University of Southampton, United Kingdom \\ \{p.rosinger,bmah\}@ecs.soton.ac.uk
}

\author{
Nicola Nicolici ${ }^{\dagger}$ \\ Dept. of ECE \\ McMaster University, Canada \\ nicola@ece.mcmaster.ca
}

\begin{abstract}
Low power design techniques have been employed for more than two decades, however an emerging problem is satisfying the test power constraints for avoiding destructive test and improving the yield. Our research addresses this problem by proposing a new method which maintains the benefits of mixed-mode built-in self-test (BIST) (low test application time and high fault coverage), and reduces the excessive power dissipation associated with scan-based test. This is achieved by employing dual linear feedback shift register (LFSR) re-seeding and generating mask patterns to reduce the switching activity. Theoretical analysis and experimental results show that the proposed method consistently reduces the switching activity by $25 \%$ when compared to the traditional approaches, at the expense of a limited increase in storage requirements.
\end{abstract}

\section{Introduction}

Scan-based built-in self-test (BIST) represents an attractive solution to the challenges of testing complex integrated circuits. Using simple built-in structures for pattern generation and test response analysis eliminates the need for expensive external test equipment as well as the problem of external access to internal components (cores) of complex integrated circuits. The general approach uses a simple random-pattern generator, for example a linear feedback shift register (LFSR), which minimizes both the hardware overhead and the impact on system's performance [1]. Several techniques have been proposed for alleviating the problem of random pattern resistant (RPR) faults. These solutions range from test point insertion to weighted random pattern testing and mixed-mode testing [1] and offer different trade-offs between fault coverage, area, performance and testing time.

In mixed-mode BIST a limited number of pseudorandom vectors are used to cover the easy-to-detect faults, while the few remaining RPR (or hard-to-detect) faults are covered with a small number of deterministic vectors. The

\footnotetext{
* Work carried out in part at McMaster University

†Research supported in part by Micronet (C.6.MM2) and NSERC (RGP239-003-01)
}

deterministic vectors are stored on-chip in a compressed format and expanded during test (i.e. "store and generate" architectures) $[5,8,6]$, or directly embedded into an LFSR sequence by "bit-fixing" [11] or "bit-flipping" [13] techniques. Although "bit-fixing" and "bit-flipping" techniques provide high quality tests, the corresponding BIST hardware is very dependent on the test set and the circuit under test (CUT), thus any change in the test set or CUT requires a complete re-synthesis of the BIST hardware. "Store and generate" BIST architectures represent a more flexible solution with comparable associated costs. In these approaches, deterministic patterns are encoded as seeds of simple test pattern generators (TPGs) such as LFSRs [6] or multiplepolynomial LFSRs (MP-LFSRs) [5, 8]. Koenemann [6] proposed an interesting method of encoding test data based on controlled re-seeding of single polynomial LFSRs. This technique is suitable for scan designs and delivers, at the cost of a small storage requirement, shorter test application time when compared to weighted random pattern generators. Hellebrand et al. [5] extended the LFSR re-seeding technique to multiple-polynomial LFSRs which reduces the storage requirements and the LFSR length when compared to the re-seeding of single-polynomial LFSRs. Rajski et. al. [8] adapted the MP-LFSR TPG to a test environment with multiple scan-chains and boundary scan chain.

While mixed-mode BIST solves the test application time and fault coverage problems associated with pseudorandom test [2], its main drawback is the excessive power dissipation caused by the uncorrelated sequences in the scan chain. The excessive power dissipation may lead to destructive test or manufacturing yield loss. Many techniques for reducing switching activity during scan or BIST have been proposed recently and summarized in $[4,7]$. The TPG schemes for generating correlated vectors [12] or non-detecting pattern suppression [3] require very little hardware overhead, however they do not provide high fault coverage for circuits with RPR faults, which is the very purpose of mixed-mode BIST. To the best of our knowledge no previous approaches have addressed the problem of high power dissipation associated with mixed-mode BIST, which is the aim of this paper.

This paper presents a new TPG architecture based on re-seeding MP-LFSR structures which achieves high fault coverage in short test application time while consistently re- 


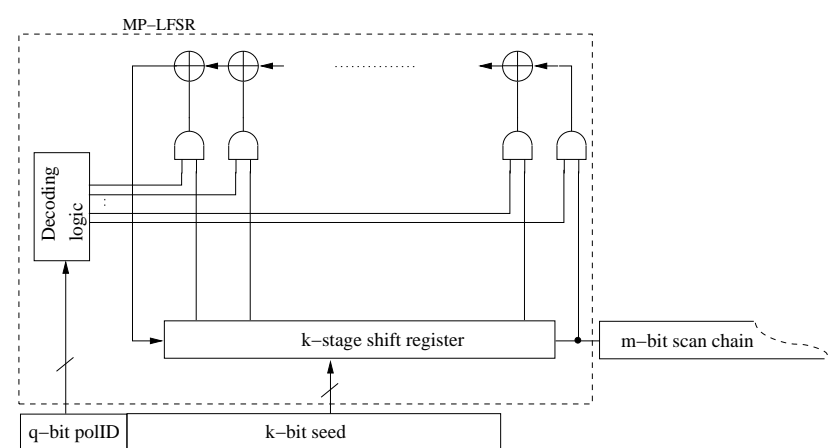

Figure 1. MP-LFSR approach for mixed-mode BIST $[5,8]$

ducing the number of transitions in the scan chain by $25 \%$. The rest of the paper is organized as follows. Section 2 reviews the basic concepts behind the use of re-seedable MPLFSRs and their high switching activity. Section 3 presents our method for reducing the number of transitions in the scan chain, and consequently the power dissipation in the circuit under test, without affecting the test quality. Our method exploits the "masking" properties of AND and OR logic functions and the randomness of patterns generated by LFSRs. Section 4 describes the TPG architecture which implements the method presented in section 3. Section 5 reports the results of the experiments performed to assess the efficiency of our method.

\section{Deterministic test generation by re-seeding multiple-polynomial LFSRs}

In this section we summarize the theory for encoding deterministic test cubes using MP-LFSRs and, using a motivational example, we show that this approach can lead to unnecessarily high switching activity.

While most faults in a circuit can be detected with high probability with any pseudo-random pattern sequence of a certain, yet reasonable, length, detecting the few RPR faults of a circuit requires prohibitively long pseudo-random sequences. Hence, in order to keep the test application time within acceptable limits, in mixed mode BIST the easy-todetect faults are covered with a limited number of LFSRgenerated pseudo-random patterns, and the remaining RPR faults are detected using a few deterministic test cubes stored on chip. The sparseness of "care bits" (or specified bits) in deterministic test cubes (typically less than $10 \%$ of the length of the scan chain) suggested the idea of encoding them into a compressed form in order to avoid large and unjustified test data storage requirements.

Figure 1 shows the basic structure of a MP-LFSR pattern generator. The decoding logic is used to select one of the several possible LFSR feedback configurations, and the seeds are loaded serially or in parallel into the shift register. Hence, in this approach, a test cube $C$ is encoded as an identifier for the LFSR feedback polynomial (the q-bit polID from Figure 1) and the initial seed (the k-bit seed from Figure 1) which will generate a test vector covered by $C$. Let $h(x)=x^{k}+\sum_{i=0}^{k-1} h_{i} x^{i}$ be the feedback polynomial of the LFSR and

$$
A(t)=\left[\begin{array}{c}
a_{0}(t) \\
\vdots \\
a_{k-1}(t)
\end{array}\right]
$$

the state of the shift register at clock $t$. The system can be described as

$$
A(t+1)=T_{S} A(t)
$$

where

$$
T_{S}=\left[\begin{array}{cccccc}
0 & 1 & 0 & \ldots & 0 & 0 \\
0 & 0 & 1 & \ldots & 0 & 0 \\
\vdots & \vdots & \vdots & & \vdots & \vdots \\
0 & 0 & 0 & \ldots & 1 & 0 \\
0 & 0 & 0 & \ldots & 0 & 1 \\
1 & h_{1} & h_{2} & \ldots & h_{k-2} & h_{k-1}
\end{array}\right]
$$

represents the state transition matrix for the given LFSR. Let $C=\left(c_{0}, \ldots, c_{m-1}\right) \in\{0,1, X\}^{m}$ be a test cube and $S(C)=\left\{i \mid c_{i} \neq X\right\}$ the set of specified bits of $C$. $C$ can be generated using the LFSR described by $T_{S}$ if the following system of linear equations:

$$
c_{i}=a_{i}=\left[T_{S}^{i} A(0)\right]_{1}, \text { for all } i \in S(C)
$$

is consistent, where $\left[T_{S}^{i} A(0)\right]_{r}$ denotes the $r$-th component of $\left[T_{S}{ }^{i} A(0)\right]$. The solution of this system of equations, if it exists, represents the initial seed from which the LFSR described by $h(x)$ will generate a test vector covered by cube $C$. It was shown in [5] that the probability of not finding a seed for a test cube with $s=|S(C)|$ specified bits using a LFSR with $s+4$ stages and 16 possible feedback polynomials is less than $10^{-6}$. Thus, by using this technique the storage requirements for encoding a test cube are determined only by the number of specified bits in the test cube. The procedure for computing the initial seed for a given test cube and feedback polynomial is illustrated in the following example.

Example 1 Consider test cube $C$ = $(X, 1,1, X, X, 0, X, X, 1, X)$ and a 4 -stage LFSR with the characteristic polynomial given by $h(x)=x^{4}+x^{3}+1$. The corresponding transition matrix will be

$$
T_{S}=\left[\begin{array}{llll}
0 & 1 & 0 & 0 \\
0 & 0 & 1 & 0 \\
0 & 0 & 0 & 1 \\
1 & 0 & 0 & 1
\end{array}\right]
$$

The equations which need to be solved in order to find the initial seed are

$$
\left[\begin{array}{c}
c_{0}=X \\
c_{1}=1 \\
c_{2}=1 \\
c_{3}=X
\end{array}\right]=T_{S}{ }^{0}\left[\begin{array}{l}
a_{0} \\
a_{1} \\
a_{2} \\
a_{3}
\end{array}\right]=\left[\begin{array}{llll}
1 & 0 & 0 & 0 \\
0 & 1 & 0 & 0 \\
0 & 0 & 1 & 0 \\
0 & 0 & 0 & 1
\end{array}\right]\left[\begin{array}{l}
a_{0} \\
a_{1} \\
a_{2} \\
a_{3}
\end{array}\right]
$$

and 


$$
\left[\begin{array}{c}
c_{5}=0 \\
c_{6}=X \\
c_{7}=X \\
c_{8}=1
\end{array}\right]=T_{S}{ }^{5}\left[\begin{array}{l}
a_{0} \\
a_{1} \\
a_{2} \\
a_{3}
\end{array}\right]=\left[\begin{array}{llll}
1 & 1 & 0 & 1 \\
1 & 1 & 1 & 1 \\
1 & 1 & 1 & 0 \\
0 & 1 & 1 & 1
\end{array}\right]\left[\begin{array}{l}
a_{0} \\
a_{1} \\
a_{2} \\
a_{3}
\end{array}\right]
$$

Solving the equations above will produce the following solution, i.e. initial seed

$$
\left[\begin{array}{l}
a_{0} \\
a_{1} \\
a_{2} \\
a_{3}
\end{array}\right]=\left[\begin{array}{l}
0 \\
1 \\
1 \\
1
\end{array}\right]
$$

The test pattern generated by the given LFSR starting from the computed initial seed will be $P=$ $(0, \underline{1}, \underline{1}, 1,1, \underline{0}, 1,0, \underline{1}, 1)$, where the underlined positions represent the specified bits from the original test cube. This example has shown how a 10-bit test cube can be encoded as a 4-bit LFSR seed. The pattern $P$ generated using this initial seed contains 5 transitions between successive bits.

Patterns generated by LFSRs, with or without re-seeding, contain a large number of transitions between consecutive bits due to the pseudo-random behavior of LFSRs. In the following section we are proposing a method for reducing the number of transitions from LFSR-generated patterns by exploiting properties of AND and OR logic functions. Our approach, tailored for a mixed-mode BIST environment, overcomes the shortcomings of previous solutions for low power scan testing by providing high fault coverage within short test application time and with reduced power dissipation during test.

\section{Reducing the number of transitions in the scan chain}

This section explains the method we are proposing in order to reduce the number of transitions in the scan chain when using a MP-LFSR based TPG. First, we give some definitions and basic concepts.

Given a logic signal $S$, the signal probability $P_{1}(S)$ represents the average fraction of clock cycles when signal $S$ is 1 . Analogously, $P_{0}(S)$ represents the average fraction of clock cycles when signal $S$ is 0 . Obviously, $P_{0}(S)+P_{1}(S)=1$. If signal $S$ is generated by a random source, its signal probabilities are equal $P_{0}(S)=P_{1}(S)=$ 0.5 The transition probability of signal $S, P_{t r}(S)$ represents the average fraction of clock cycles when the current value of $S$ is different than its previous value. Assuming temporal independence between consecutive values of $S$, the transition probability can be computed as $P_{t r}(S)=P_{0}(S) \times P_{1}(S)+P_{1}(S) \times P_{0}(S)$. The transition probability of a random logic signal $S$ is

$$
P_{t r}(S)=0.5 \times 0.5+0.5 \times 0.5=0.5
$$

Assume two mutually independent random signals $S_{A}$ and $S_{B}$, and let $S_{A N D}=S_{A} A N D S_{B}$. Given the mutual independence of $S_{A}$ and $S_{B}$, the signal probabilities of $S_{A N D}$ can be computed as follows:

$$
\begin{aligned}
& P_{1}\left(S_{A N D}\right)=P_{1}\left(S_{A}\right) \times P_{1}\left(S_{B}\right)=0.25 \\
& P_{0}\left(S_{A N D}\right)=1-P_{1}\left(S_{A N D}\right)=0.75
\end{aligned}
$$

Hence, the transition probability of $S_{A N D}$ is given by

$$
\begin{aligned}
P_{t r}\left(S_{A N D}\right) & =2 \times P_{1}\left(S_{A N D}\right) \times P_{0}\left(S_{A N D}\right) \\
& =0.375
\end{aligned}
$$

In a similar fashion we can compute the transition probability of an OR composition of $S_{A}$ and $S_{B}, S_{O R}=$ $S_{A} O R S_{B}$

$$
P_{t r}\left(S_{O R}\right)=0.375
$$

From equations (2), (3) and (4) we conclude that both AND and OR compositions of two mutually independent random signals produce signals with transition probabilities $25 \%$ lower than those of the original signals. The fact that LFSR-generated bit-sequences exhibit a high degree of randomness together with the previous observation motivated the use of AND/OR composition for reducing the number of transitions in the scan chain, and consequently the power dissipation in the CUT. In the following we will explain how this method can be used in a mixed mode BIST environment. There are two problems which need to be addressed in order to guarantee the fault coverage of patterns generated by AND/OR composition:

- The first problem is to ensure that by AND/OR composition we can produce patterns with good random properties for covering the easy-to-detect faults within a reasonable amount of time. This is achieved by performing the AND/OR composition on mutually independent patterns.

- The second problem is to employ AND/OR composition for generating patterns corresponding to deterministic test cubes for RPR faults. This is addressed next.

The previous section has shown how a pattern $P$ covering the specified bits in a test cube $C$ can be generated by re-seeding an LFSR. What we need now is an algorithmic method for deriving a "mask pattern" $M P$ which, by AND/OR composition with $P$, will produce a pattern $P^{\prime}$ which covers the specified bits from $C, S(C)=\{i \in$ $\left.C \mid c_{i} \neq X\right\}$. The procedure we are proposing for generating the suitable"mask pattern" consists of the following steps:

1. chose a composition function $f_{\text {comp }} \in\{A N D, O R\}$

2. from $C$ compute the "mask cube" $M C$ comprising the bits in $M(C)=\left\{i \in S(C) \mid c_{i}=N C V\left(f_{\text {comp }}\right)\right\}$, where $N C V\left(f_{\text {comp }}\right)$ is the non-controlling value of $f_{\text {comp }}$, i.e. 1 for AND and 0 for OR;

3. generate a "mask pattern" $M P$ which covers the specified bits in $M(C)$. 
The $M P$ constructed using the above procedure guarantees that the pattern $P^{\prime}=f_{\text {comp }}(P, M P)$ will cover all the specified bits from $C$. This is because the bits in $M(C)$ are covered both by $P$ and $M P$, and hence they are preserved through $f_{\text {comp }}$, while the bits in $S(C)-M(C)$ are covered by $P$ and having the controlling value of $f_{\text {comp }}$ they are not altered by $f_{\text {comp }}$. Thus, both $P$ and $M P$ can be generated by seeding LFSR structures. The seeds for $P$ and $M P$ can be computed as described in the previous section. The following example illustrates the procedure for generating the "mask pattern" for a given test cube.

Example 2 Assume we want to generate the "mask pattern" $M P$ corresponding to the AND composition for the deterministic cube $C=(X, 1,1, X, X, 0, X, X, 1, X)$ from Example 1. The "mask cube" for AND composition is $M C=(X, 1,1, X, X, X, X, X, 1, X)$. Consider $h_{m}(x)=$ $x^{3}+x+1$ as the characteristic polynomial LFSR we will use to generate $M P$. We compute the initial seed for $M C$ as described in the previous section. The state transition matrix associated to the LFSR is:

$$
T_{S m}=\left[\begin{array}{lll}
0 & 1 & 0 \\
0 & 0 & 1 \\
1 & 1 & 0
\end{array}\right]
$$

The equations needed to compute the initial seed are:

$\left[\begin{array}{c}c_{0}=X \\ c_{1}=1 \\ c_{2}=1\end{array}\right]=T_{S_{m}} 0\left[\begin{array}{l}a_{0} \\ a_{1} \\ a_{2}\end{array}\right]=\left[\begin{array}{lll}1 & 0 & 0 \\ 0 & 1 & 0 \\ 0 & 0 & 1\end{array}\right]\left[\begin{array}{l}a_{0} \\ a_{1} \\ a_{2}\end{array}\right]$

and

$$
\left[\begin{array}{c}
c_{6}=X \\
c_{7}=X \\
c_{8}=1
\end{array}\right]=T_{S m}{ }^{6}\left[\begin{array}{l}
a_{0} \\
a_{1} \\
a_{2}
\end{array}\right]=\left[\begin{array}{lll}
1 & 0 & 1 \\
1 & 0 & 0 \\
0 & 0 & 1
\end{array}\right]\left[\begin{array}{l}
a_{0} \\
a_{1} \\
a_{2}
\end{array}\right]
$$

Solving these equations will lead to the following seed:

$$
\left[\begin{array}{l}
a_{0} \\
a_{1} \\
a_{2}
\end{array}\right]=\left[\begin{array}{l}
1 \\
1 \\
1
\end{array}\right]
$$

The "mask pattern" which will be generated using this seed is $M P=(1, \underline{1}, \underline{1}, 0,0,1,0,1, \underline{1}, 1)$, where the underlined positions represent the specified bits from $M C$. The pattern resulted by AND composition of $P$ and $M P$ is $P^{\prime}=(0, \underline{1}, \underline{1}, 0,0, \underline{0}, 0,0, \underline{1}, 1)$, where the underlined positions show the specified bits from the original test cube $C$, preserved through AND composition. The pattern $P$ from Example 1 generated by the traditional MP-LFSR TPG has 5 transitions between consecutive bits, while the pattern $P^{\prime}$ produced by our method for the same deterministic cube contains only 3 transitions between consecutive bits.

Thus we have shown how suitable "mask patterns" which reduce the number of transitions in the scan chain by AND (OR) composition can be generated in a similar manner with patterns for deterministic test cubes. By using our method, a deterministic cube is encoded as two (polynomial identifier, initial seed) pairs: one pair encodes the deterministic test cube while the second pair encodes the "mask cube", corresponding to the test cube and to the selected composition function, which ensures that the specified bits in the test cube are preserved through composition. The following section describes a new TPG architecture which implements this method.

\section{Test pattern generator for low power mixed- mode BIST}

In the previous section we have shown how $\mathrm{AND}(\mathrm{OR})$ composition of MP-LFSR generated pseudo-random sequences can be used to reduce the number of transitions in the scan chain during pseudo-random test. We have also provided a method of generating "mask patterns" for reducing the number of transitions during the deterministic testing mode without affecting the fault coverage. In this section we will describe the architecture of our TPG based on AND(OR) composition and re-seeding of MP-LFSRs.

The basic idea is to have two different MP-LFSRs operated in parallel and apply to the input of the scan chain the AND/OR composition of the bit-sequences generated by the two MP-LFSRs. The main MP-LFSR will act as a traditional MP-LFSR TPG [5, 8], while the secondary MPLFSR will produce "mask patterns" to reduce the number of transitions in the scan chain. We have implemented this solution with the TPG shown in Figure 2. The two MP-LFSRs share the decoding logic for selecting the feedback configurations in order to minimize the hardware overhead and the memory required for storing the polynomial identifiers for the test and mask cubes. The randomness of the test patterns generated by such an architecture, which ensures rapid coverage of easy-to-detect faults, can be easily achieved by using different primitive characteristic polynomials for the two MP-LFSRs. Generation of deterministic patterns for covering the RPR faults is achieved by re-seeding the two MP-LFSRs with stored seeds computed as explained in sections 2 and 3.

The minimum length of the MP-LFSR, and consequently the size of the memory for storing the initial seeds, is given by the number of specified bits in the cube to be encoded, as mentioned in section 2. The size of the main MP-LFSR, $k$ from Figure 2, is determined by the maximum number of specified bits per cube in the precomputed test cubes. However in the case of the secondary MP-LFSR, for each test cube we have two possible mask cubes, one for AND composition and one for OR composition. We can exploit this fact by selecting for each test cube the composition function which leads to the mask cube with less specified vectors. The $M S$ bit from Figure 2 is used to select the appropriate composition function for each test cube. In the following we will determine an upper bound for the maximum number of specified bits in mask cubes when we can choose for each cube between AND and OR composition. Given a test cube $C$, let $M_{A N D}(C)$ and $M_{O R}(C)$ be the sets of specified bits of the mask cubes corresponding to AND and OR composition respectively. The mask cube which will be selected in order to minimize the MP-LFSR size will have 


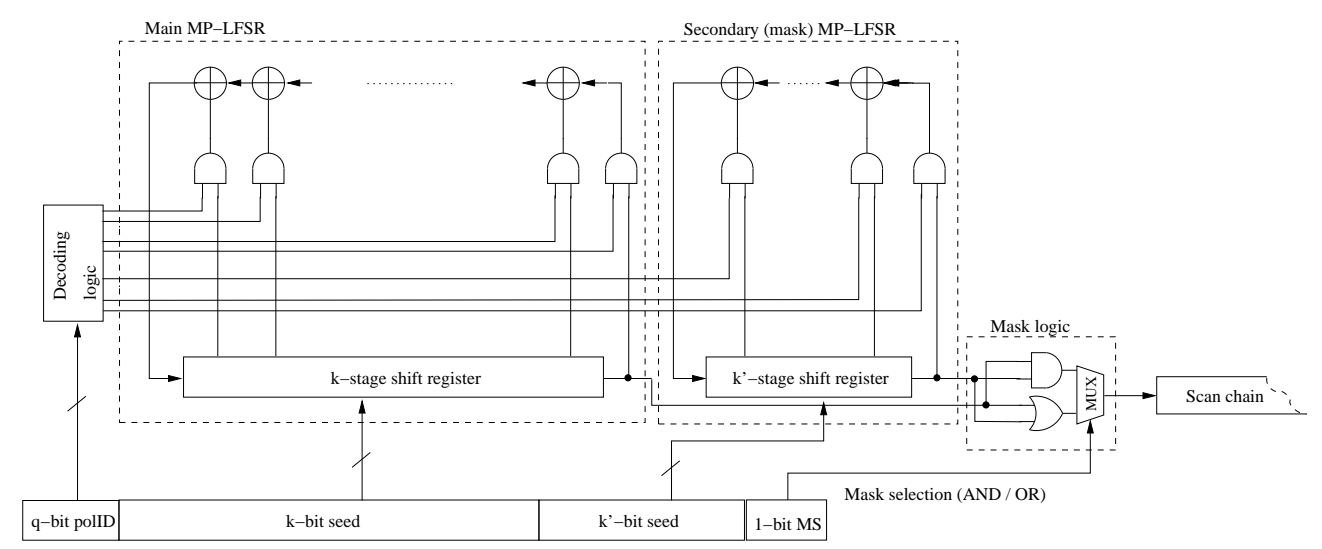

Figure 2. Proposed dual MP-LFSR TPG architecture for low power mixed-mode BIST

$s^{\prime}=\min \left(\left|M_{A N D}(C)\right|,\left|M_{O R}(C)\right|\right)$ specified bits. As the sets of specified bits of $M_{A N D}(C)$ and $M_{O R}(C)$ are disjoint, and their union is equal to $S(C)$, the set of specified bits of $C$, we have

$$
\begin{aligned}
\left|M_{A N D}(C)\right| & +\left|M_{O R}(C)\right|=|S(C)| \\
s^{\prime} & \leq\left|M_{A N D}(C)\right| \\
s^{\prime} & \leq\left|M_{O R}(C)\right|
\end{aligned}
$$

By adding inequalities (6) and (7) and using equation (5) we obtain:

$$
s^{\prime} \leq \frac{|S(C)|}{2}
$$

Thus the upper bound for the length of the secondary MPLFSR, $k^{\prime}$ from Figure 2, is given by $\frac{|S(C)|}{2}+l$, where $l$ is a small constant, 2 in our experiments, which ensures that the probability of finding an initial seed for each mask cube is high, and consequently the total number of feedback polynomials is small. Although intuitively it seems that we need to double the storage requirements, inequality (8) has shown that the length of the secondary (mask) MP-LFSR from Figure 2 is only approximately $50 \%$ of the length of the main MP-LFSR, whose length is dependent on $|S(C)|$ (see section 2).

\section{Experimental results}

We have performed several experiments using the full scan versions of the ISCAS-89 circuits in order to asses the efficiency and the cost of the proposed method. We have used ATALANTA [9] for automatic generation of the deterministic test cubes and FSIM [10] for fault simulation. The method for computing initial seeds and feedback polynomials described in section 2 , and our method for deriving mask patterns described in section 3 were implemented using $\mathrm{C}++$.

In our experiments shown in Table 1, we have first assigned some default initial seeds and feedback polynomials to the two MP-LFSRs. We have also selected a default composition function, AND for example. Using this configuration we have generated $1 \mathrm{k}$ pseudo-random patterns and

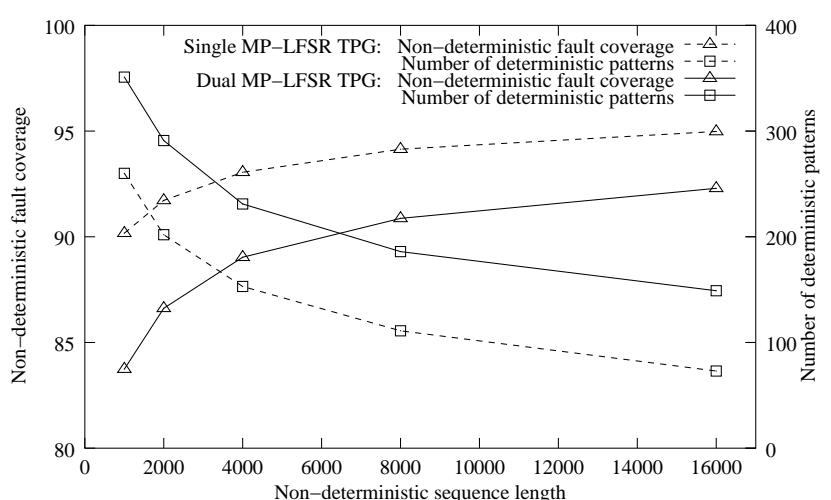

Figure 3. Circuit s38584: Non-deterministic fault coverage vs. the number of deterministic patterns for single and dual MP-LFSR TPG

fault simulated them on the target circuit. For the remaining undetected faults we have used ATALANTA to derive the deterministic test cubes. We have set the length of the main MP-LFSR to $P D m=s_{\max }+2$ where $s_{\max }$ is the maximum number of specified bits $s$ in all the test cubes in the precomputed test cube set. We have set the length of the secondary MP-LFSR to $P D s=s_{\text {max }}^{\prime}+2$ where $s_{\text {max }}^{\prime}$ is the maximum number of specified bits $s^{\prime}$ in all the mask cubes. Having set the length of both MP-LFSRs, we have computed for each test cube and corresponding mask cube the initial seeds, feedback polynomials and corresponding output patterns for the two MP-LFSRs as well as the pattern resulted from the composition of the latter. Column NC from Table 1 gives the number of deterministic test cubes computed to achieve maximal fault coverage. Columns NPm and NPs show the number of polynomials used by the main and secondary MP-LFSRs to cover all test and mask cubes. Columns PDm and PDs give the length of the polynomials used by the two MP-LFSRs (main and secondary). Columns TC and origTC give the number of transitions in the scan chain obtained by using our TPG and respectively a traditional TPG with a single MP-LFSR [5, 8]. Column redTC\% shows the reduction in transition count, which is 


\begin{tabular}{|c|c|c|c|c|c|c|c|c|c|c|c|}
\hline Circuit & NC & NPm & PDm & NPs & PDs & TC & origTC & redTC\% & xStorage\% & origFC\% & mFC\% \\
\hline \hline s420 & 44 & 2 & 22 & 1 & 8 & 231378 & 306721 & 24.56 & 36.36 & 76.92 & 72.3 \\
\hline s526 & 32 & 2 & 15 & 1 & 8 & 116369 & 152027 & 23.46 & 53.33 & 95.49 & 86.99 \\
\hline s641 & 23 & 1 & 24 & 2 & 10 & 577057 & 753730 & 23.44 & 41.67 & 96.32 & 88.98 \\
\hline s713 & 22 & 2 & 24 & 2 & 12 & 573862 & 753044 & 23.79 & 50 & 90.53 & 84.3 \\
\hline s820 & 53 & 5 & 15 & 1 & 8 & 108245 & 143475 & 24.55 & 53.33 & 91.41 & 81.52 \\
\hline s832 & 52 & 4 & 15 & 1 & 8 & 108090 & 143284 & 24.56 & 53.33 & 89.88 & 79.66 \\
\hline s838 & 131 & 1 & 38 & 1 & 8 & 904106 & 1202210 & 24.8 & 21.05 & 58.75 & 59.39 \\
\hline s953 & 39 & 1 & 17 & 3 & 9 & 404086 & 532909 & 24.17 & 52.94 & 83.31 & 91.47 \\
\hline s1196 & 124 & 1 & 19 & 4 & 9 & 227156 & 294182 & 22.78 & 47.37 & 88.64 & 73.91 \\
\hline s1238 & 130 & 1 & 19 & 4 & 9 & 228405 & 295494 & 22.7 & 47.37 & 83.1 & 68.48 \\
\hline s1423 & 28 & 1 & 28 & 2 & 13 & 1611456 & 2137027 & 24.59 & 46.43 & 96.96 & 93.33 \\
\hline s5378 & 199 & 2 & 29 & 5 & 15 & 10188661 & 13592997 & 25.04 & 51.72 & 93.78 & 86.17 \\
\hline s9234 & 243 & 2 & 53 & 2 & 27 & 14152373 & 18707043 & 24.35 & 50.94 & 70.72 & 63.7 \\
\hline s13207 & 319 & 3 & 24 & 3 & 13 & 120663103 & 161446001 & 25.26 & 54.17 & 79.82 & 81.14 \\
\hline s15850 & 238 & 3 & 40 & 2 & 21 & 86268594 & 115238803 & 25.14 & 52.5 & 86.23 & 84.65 \\
\hline s38417 & 498 & 3 & 88 & 2 & 45 & 771889958 & 1027848936 & 24.9 & 51.14 & 86.19 & 84.89 \\
\hline s38584 & 351 & 4 & 56 & 2 & 29 & 543841106 & 722223634 & 24.7 & 51.79 & 90.32 & 83.74 \\
\hline
\end{tabular}

Table 1. Experimental results for $1 \mathrm{k}$ pseudo-random patterns and deterministic patterns for maximal fault coverage

consistent with the probabilistic estimation of $25 \%$ reduction from section 3. Column xStorage \% shows the amount of additional storage required for storing the mask seeds, relative to the storage size for the test seeds, result which is consistent with the theoretical upper bound of nearly $50 \%$ determined in section 4. Finally, columns origFC and $\mathbf{~ m F C}$ compare the fault coverage of $1 \mathrm{k}$ pseudo-random pattern sequences and $1 \mathrm{k}$ pattern sequences generates using our TPG in non-deterministic mode. The lower fault coverage of the non-deterministic sequences generated by our TPG, a side effect of the masking process, is compensated by a higher number of deterministic patterns, needed to achieve maximal fault coverage. Figure 3 shows, for both the traditional TPG with a single MP-LFSR as well as for our dual MP-LFSR TPG, the relation between the number of nondeterministic test patterns, their fault coverage and the number of deterministic test patterns needed to achieve maximal fault coverage. The number of deterministic patterns for full fault coverage decreases as the length of the nondeteterministic sequence increases. Hence, the storage requirements can be controlled by varying the length of the non-deterministic sequence.

\section{Conclusions}

In this paper we have proposed a new TPG for mixedmode BIST based on mask pattern generation and reseeding of dual MP-LFSRs. By employing AND and OR masking, our TPG, at the cost of additional, yet limited, storage requirements, reduces the number of transitions in the scan chain by $25 \%$ while preserving the fault coverage and test application time when compared with a traditional TPG with a single MP-LFSR [5, 8]. Future work will investigate the trade-offs in power dissipation and storage requirements when using AND/OR composition functions which have an increased fan-in and hence additional mask MP-LFSRs that will further lower power dissipation during test.

\section{References}

[1] M. Abramovici, M. A. Breuer, and A. D. Friedman. Digital Systems Testing and Testable Design. IEEE Press, 1990.

[2] P. H. Bardell, W. H. McAnney, and J. Savir. Built-In Self Test - Pseudorandom Techniques. John Wiley \& Sons, 1986.

[3] S. Gerstendorfer and H. Wunderlich. Minimized power consumption for scan-based BIST. In Proc. IEEE International Test Conference, pages 77-84, 1999.

[4] P. Girard. Low power testing of VLSI circuits: Problems and solutions. In First International Symposium on Quality of Electronic Design (ISQED), pages 173-180, 2000.

[5] S. Hellebrand, J. Rajski, S. Tarnick, S. Venkataraman, and B. Courtois. Built-in test for circuits with scan based on reseeding of multiple-polynomial linear feedback shift registers. IEEE Transactions on Computers, 44(2):223-233, February 1995.

[6] B. Koenemann. LFSR-coded test patterns for scan designs. In European Test Conference, pages 237-242, 1991.

[7] N. Nicolici. Power Minimisation Techniques for Testing Low Power VLSI Circuits. PhD thesis, University of Southampton, UK, http://www.bib.ecs.soton.ac.uk/records/4937/, October 2000

[8] J. Rajski, J. Tyszer, and N. Zacharia. Test data decompression for multiple scan designs with boundary scan. IEEE Transactions on Computers, 47(11):1188-1200, November 1998.

[9] V. Tech. ATALANTA. In http://www.ee.vt.edu/ "ha/ cadtools/ cadtools.html.

[10] V. Tech. FSIM. In http://www.ee.vt.edu/"ha/ cadtools/cadtools.html.

[11] N. A. Touba and E. J. McCluskey. Altering a pseudo-random bit sequence for scan-based BIST. In IEEE Internatinal Test Conference (ITC), pages 167-175, 1996.

[12] S. Wang and S. K. Gupta. LT-RTPG: A new test-per-scan BIST TPG for low heat dissipation. In Proc. IEEE International Test Conference, pages 85-94, 1999.

[13] H. J. Wunderlich and G. Kiefer. Bit-flipping BIST. In International Conference on Computer Aided Design (ICCAD), 1996. 